\title{
Relationships between Spinal Sarcopenia and Spinal Sagittal Balance in Older Women
}

\author{
Dong Hyun Kim ${ }^{1}$, Sang Yoon Lee ${ }^{2}$, Sang Joon Park ${ }^{3}$, Young-Seok Lee ${ }^{4}$ \\ ${ }^{1}$ Department of Radiology, SMG-SNU Boramae Medical Center, Seoul National University College of Medicine, Seoul, Korea \\ ${ }^{2}$ Department of Rehabilitation Medicine, SMG-SNU Boramae Medical Center, Seoul National University College of Medicine, Seoul, Korea \\ ${ }^{3}$ Department of Radiology, Seoul National University Hospital, Seoul, Korea \\ ${ }^{4}$ Department of Neurosurgery, Gyeongsang National University Hospital, Jinju, Korea
}

Corresponding Author:

Sang Yoon Lee, $\mathrm{MD}, \mathrm{PhD}$

Department of Rehabilitation

Medicine, SMG-SNU Boramae

Medical Center, Seoul National

University College of Medicine, 20

Boramae-ro 5-gil, Dongjak-gu, Seoul

07061, Korea

E-mail: 1sy126@snu.ac.kr

ORCID:

https://orcid.org/0000-0002-2906-3094

Received: August 9, 2019

Revised: September 4, 2019

Accepted: September 11, 2019
Background: Spinal sarcopenia is receiving renewed attention as a cause of spinal sagittal imbalance. However, the relationships between spinal sarcopenia and spinal sagittal balance (SSB) have not been thoroughly investigated. We evaluated the relationships between SSB parameters and sarcopenic indices with lumbar paraspinal muscle (LPM) quantity and strength in healthy older adults. Methods: Twenty-four healthy community-dwelling older women were enrolled. Demographic variables, conventional sarcopenic indices, isometric back muscle strength, and SSB parameters, as well as results of functional examinations and lumbar spine computed tomography scan with LPM cross-sectional area (CSA) and density assessments, were examined. The independent effect on the sum of the total LPM CSA was determined using multivariable regression analysis adjusted for age, appendicular skeletal muscle mass, gait speed, handgrip strength, back extensor strength, and pelvic tilt (PT) angle. Results: PT angle was significantly correlated with the sum of the total LPM CSA and mean LPM density $(r=-0.502, p=0.015$ and $r=0.504$, $p=0.014$, respectively). Furthermore, PT angle was an independent factor for the sum of the total LPM CSA ( $\beta=-0.610, p=0.021)$ in the multivariate regression models $\left(R^{2}=0.320\right)$. Conclusion: Our data suggest that PT angle was significantly correlated with LPM CSA in healthy older women. To our knowledge, this is the first report to investigate the relationships of sarcopenic indices and spinal muscle degeneration with SSB.

Key Words: Sarcopenia, Spine, Paraspinal muscles, Lumbosacral region, Kyphosis

\section{INTRODUCTION}

The spine is an inevitable site of sarcopenia owing to the large muscles surrounding it. Sarcopenia of the lumbar paraspinal muscles (LPMs) has been receiving renewed attention as a cause of spinal degeneration. Both atrophy and fatty changes in paraspinal muscles originating from sarcopenia are associated with functional disorders and chronic back pain. ${ }^{1)}$

Conventional indices to define sarcopenia-appendicular skeletal muscle mass (ASM), handgrip strength (HGS), and gait speed-cannot reflect regional sarcopenia and its clinical outcomes. A cross-sectional study of 821 subjects with knee osteoarthritis and 4,103 controls showed that low skeletal muscle mass in the lower limbs was correlated with the presence of knee osteoarthritis, whereas whole-body skeletal muscle mass was not. ${ }^{2)}$ The authors suggested the need for limb-specific muscle mass examinations to assess the effects of skeletal muscles on a specific joint. Therefore, regional measurements should be performed to evaluate the outcome of sarcopenia in focal areas.

However, there are few simple and clinically valid measuring tools to assess sarcopenia in the spine. Whole-body dual-energy $\mathrm{X}$-ray absorptiometry and bioimpedance analysis (BIA) to measure ASM cannot be applied to spinal sarcopenia; thus, tomographic imaging such as computed tomography (CT) and magnetic resonance imaging (MRI) are required to measure paraspinal muscle quantity. Moreover, tools to verify the function and perfor- 
mance of paraspinal muscle are more difficult to evaluate. To assess paraspinal muscle strength, expensive special equipment such as isokinetic dynamometry is required. Furthermore, there is no standardized test for spinal muscle performance.

Spinal sagittal balance (SSB) is an important indicator of outcomes of lumbar spine surgery ${ }^{3)}$ and non-operative treatment of spinal stenosis. ${ }^{4)}$ While SSB can be affected by sex ${ }^{5}$ and ethnicity, ${ }^{6)}$ aging itself is the most important cause of spinal sagittal imbalance. ${ }^{7}$ LPM degeneration is one of the causes of spinal sagittal imbalance. An MRI-based study reported the relationship between the estimated LPM volume and sagittal curvature magnitude. ${ }^{8)}$ One cross-sectional study suggested that the muscle thickness of the erector spinae and echo intensity of the lumbar multifidus were independent variables of SSB. ${ }^{1 \text { ) }}$

However, the relationships between spinal sarcopenia and SSB have not been thoroughly investigated. Thus, we evaluated the relationships of SSB parameters and sarcopenic indices with LPM quantity and strength in healthy older adults. We hypothesized that SSB parameters could reflect LPM mass and back extensor strength.

\section{MATERIALS AND METHODS}

\section{Study Population}

Healthy community-dwelling older ( $\geq 65$ years) women who could independently walk more than $100 \mathrm{~m}$ were consecutively enrolled in a single center from July 2018 to December 2018. Participants who had experienced the following were excluded: (1) low back pain with moderate severity (numeric rating scale 5 and over); (2) history of any type of lumbar spine surgery; (3) history of hip fracture surgery and arthroplasty of the hip or knee; (4) disorders of the central nervous system such as stroke, parkinsonism, or spinal cord injury; (5) communication disorder such as severe hearing loss; (6) musculoskeletal conditions affecting physical function such as limb amputation; (7) long-term use of corticosteroids due to inflammatory disease; (8) malignancy requiring treatment within 5 years; and (9) other medical conditions requiring active treatment; additionally, individuals who refused to participate in the study were also excluded.

\section{Conventional Sarcopenia Work-Up}

BIA (InBody 720; InBody, Seoul, Korea) was used to analyze body composition including lean body and fat masses. ASM was calculated as the sum of the lean mass in the bilateral upper and lower extremities ${ }^{9)}$ and standardized by dividing by the squared height $\left(\mathrm{ASM} / \mathrm{Ht}^{2}, \mathrm{~kg} / \mathrm{m}^{2}\right)$. HGS was measured using a hand-grip dynamometer (T.K.K.5401; Takei Scientific Instruments, Tokyo, Japan), ${ }^{10)}$ as described previously. ${ }^{11)}$ Briefly, participants were asked to perform the following while sitting in a straight-backed chair with their feet flat on the floor: adduct and neutrally rotate the shoulder, flex the elbow to $90^{\circ}$, and place the forearm in a neutral position with the wrist between $0^{\circ}$ and $30^{\circ}$ extension and between $0^{\circ}$ and $15^{\circ}$ ulnar deviation. Participants were instructed to squeeze the handle as hard as possible for 3 seconds, and the maximum contraction force $(\mathrm{kg})$ was recorded. Gait speed was measured using a 6-meter usual gait speed $(\mathrm{m} / \mathrm{s})$ as recommended by the Asian Working Group for Sarcopenia. The definition and cutoffs for sarcopenia were also adopted from these guidelines. ${ }^{12)}$

\section{Functional Examinations and Questionnaires}

Functional examination using a short physical performance battery (SPPB) was derived from three objective physical function tests (i.e., time taken to cover $4 \mathrm{~m}$ at a comfortable walking speed, time taken to stand from sitting in a chair five times without stopping, and ability to maintain balance for 10 seconds in three different foot positions at progressively more challenging levels). ${ }^{13)} \mathrm{A}$ score from 0 to 4 was assigned to the performance of each task, with higher scores indicating better lower body function. The Timed Up and Go test (TUG) has shown excellent test-retest reliability in older adults. ${ }^{14)}$ The participants were provided with verbal instructions to stand up from an armchair, walk $3 \mathrm{~m}$ as fast as possible, turn back at a cone set out by the researchers, walk back, and sit down in the chair. They were allowed to wear their regular footwear and use a walking aid if needed. A stopwatch was started on the word 'go' and stopped when the participant was completely seated with their back against the backrest. The time to complete the test was recorded in three consecutive trials using the first trial to familiarize the participants with the test. The best time from the three trials was analyzed. ${ }^{15)}$ The Oswestry Disability Index (ODI) is one of the most commonly used instruments for measuring disability in spinal disorders. It consists of 10 items that assess the level of pain and interference with several physical activities. We used the Korean version of the ODI. ${ }^{16)}$ The Back Performance Scale (BPS) consists of five tests: sock test, pick-up test, roll-up test, fingertip-to-floor test, and lift test. The five tests comprising the BPS demonstrate associations with each other and each test contributes to the high internal consistency, implying that the tests share a common characteristic in measuring physical performance. ${ }^{17)}$ The BPS sum score (0-15) is calculated by adding the individual scores for the five tests.

\section{Isometric Back Muscle Strength}

We measured the isometric back extensor strength using a handheld dynamometer (PowerTrack II; JTECH Medical, Salt Lake City, UT, USA) ${ }^{18)}$ Briefly, the participants stood in full extension 
with their backs to a wall and feet flat on the floor with heels touching the wall. An inelastic belt was looped through the anchor rails and secured firmly $1 \mathrm{~cm}$ below the anterior superior iliac spine to restrain movement and maintain participant contact with the wall during the test. The participants were instructed to flex forward approximately $15^{\circ}$ at the hips so that the dynamometer could be positioned posterior to the spinous process of the 7 th thoracic vertebrae. In this way, counter pressure was provided by the fixed wall behind the participants' back to avoid tester-induced variations in resistance. Although this method is novel, it showed a strong positive relationship with back extensor strength measured using the gold-standard isokinetic dynamometry and high inter-instrument validity and reliability. ${ }^{19)}$

\section{Spinal Sagittal Balance}

For each patient, one lateral radiograph of the whole spine was obtained and digitized. All measurements were performed using imaging software (INFINITT PACS M6; INFINITT Healthcare, Seoul, Korea), as previously described. ${ }^{20,21)}$ The following spinopelvic radiographic parameters were analyzed: sacral slope (SS), pelvic incidence (PI), pelvic tilt (PT), lumbar lordosis (LL), thoracic kyphosis (TK), and sagittal vertical axis (SVA).

\section{LPM Measurement by Spine CT Scan}

Lumbar spine CT scans (Ingenuity CT; Philips Healthcare, Cleveland, $\mathrm{OH}, \mathrm{USA}$ ) were performed to measure the cross-sectional area (CSA) and mean density (in Hounsfield unit [HU]) of the LPM (multifidus $[\mathrm{MF}]$ and erector spinae $[\mathrm{ES}])^{22)}$ The mean den- sity reflected the degree of intramuscular fat content because the values decreased as the fat content increased. Before each CT scan, a calibration was performed using air as the standard. CT scanning was performed with the participant in the supine position with a $120-\mathrm{kV}$ and 140-mA protocol. Using 1-mm thin-section axial CT scan images, the axial images were reformatted with each lumbar intervertebral disc level (T12/L1-L5/S1) parallel to the adjacent vertebral endplates. These axial images at each intervertebral disc level were reconstructed at 2.5 -mm intervals, which included cross-sectional images of LPM. The measurement of ES and MF was performed from the level of L1/L2 to L5/S1 using a specially designed radiological workstation (MEDIP; MEDICALIP, Seoul, Korea) (Fig. 1). The CSA was measured by manually constructing points around the outer margins of the individual muscles using a touchscreen LCD monitor (XPS 15 9570; Dell Inc., Round Rock, TX, USA) and digital touchscreen pen (PN556W Dell Active Pen; Dell Inc.). After the CSA and mean density of paraspinal muscles were separately measured on the bilateral sides, the mean and sum values at all levels were calculated. ${ }^{23)}$

All demographic and clinical data, including CT scan images, were obtained with the approval of the Institutional Review Board of SMG-SNU Boramae Medical Center (No. 16-2017-45). Written informed consent was obtained from all participants.

\section{Statistical Analysis}

The relationships between sarcopenic indices and functional outcomes with SSB parameters were measured by Pearson correlation coefficients. The independent effects on the sum of the total LPM
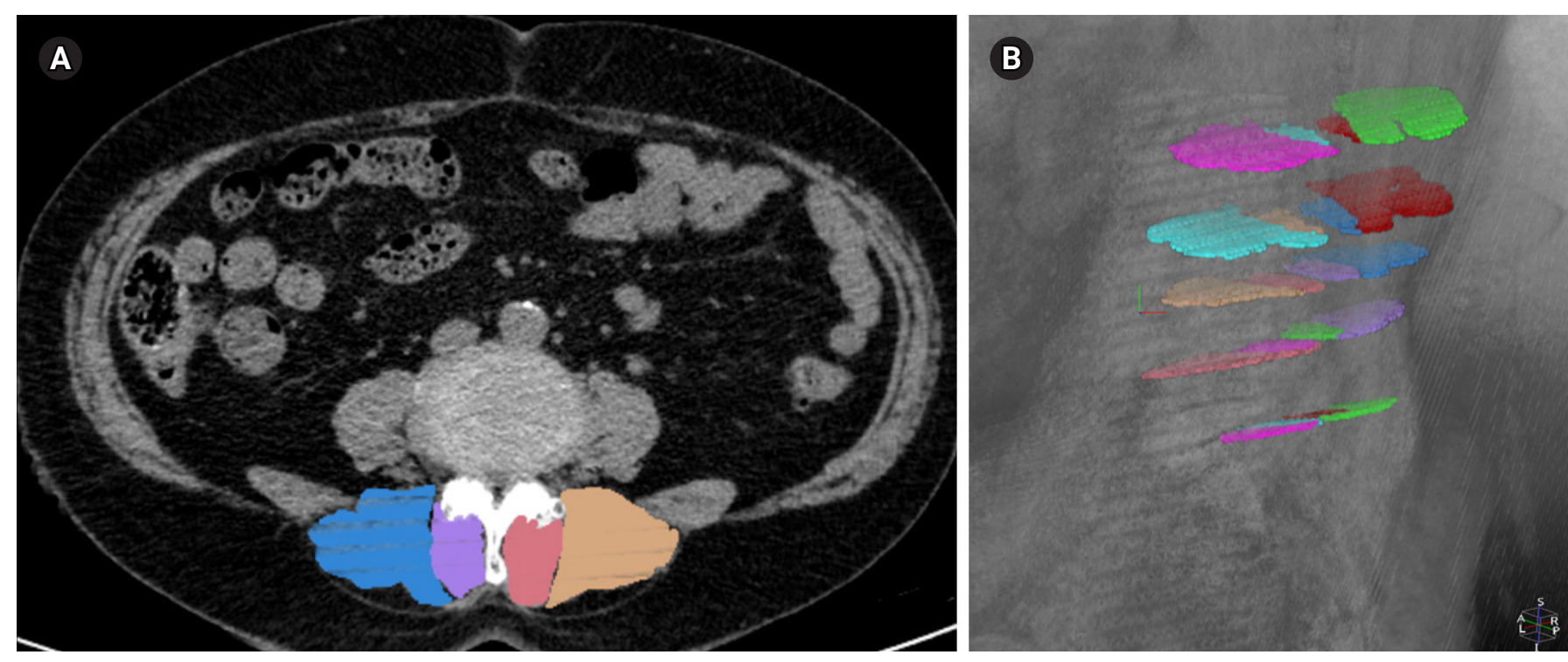

Fig. 1. Measurements of cross-sectional area and density of lumbar paraspinal muscle. (A) Computed tomography axial image at the L3/4 disc level and (B) three-dimensional reconstructed bird's-eye view from the top-left of the participant. 
CSA were determined using multivariate regression models adjusted for six key factors: age, ${ }^{7)}$ ASM/ $\mathrm{Ht}^{2}$, HGS, gait speed, ${ }^{1)}$ back extensor strength, ${ }^{24)}$ and PT angle. ASM/ $\mathrm{Ht}^{2}$ and HGS were included because they are the basic variables for diagnosing sarcopenia and PT was included in the regression analysis as the most relevant variable of SSB. An adjusted model was developed through backward elimination with a significance level of 0.2 to enter and 0.05 to retain. We also evaluated possible multiple collinearities between covariates by correlation analysis and collinearity statistical tests (tolerance and variance inflation factor tests) during regression analysis. IBM SPSS Statistics version 21.0 for Windows (IBM Corp., Armonk, NY, USA) was used for all analyses. p-values of less than 0.05 were considered statistically significant.

\section{RESULTS}

The baseline characteristics of 24 older women are shown in Table 1. Their mean age was $76.8 \pm 4.2$ years. Among conventional sarcopenic indices, the ALM $/ \mathrm{Ht}^{2}, \mathrm{HGS}$, and gait speed were $6.30 \pm 0.79$ $\mathrm{kg} / \mathrm{m}^{2}, 20.0 \pm 3.4 \mathrm{~kg}$, and $0.87 \pm 0.16 \mathrm{~m} / \mathrm{s}$, respectively. No participant met the diagnostic criteria for sarcopenia.

Among the conventional sarcopenic indices, only HGS was positively correlated with SPPB $(r=0.521, \mathrm{p}=0.011)$. While both HGS and $\mathrm{ASM} / \mathrm{Ht}^{2}$ tended to have positive correlations with LPM mean density and back extensor strength, they were not significant (Table 2). Among SSB parameters, PT angle was significantly correlated with the sum of the total LPM CSA and mean LPM density ( $r=-$ $0.502, p=0.015$ and $r=0.504, p=0.014$, respectively) (Fig. 2 ). The $\mathrm{LL}$ angle was also correlated with the ODI and the sum of the total LPM CSA $(r=-0.423, p=0.045$ and $r=0.439, p=0.036$, respectively). Only PI angle was significantly correlated with back extensor strength $(\mathrm{r}=-0.490, \mathrm{p}=0.018)$ (Table 3).

Finally, among the six key variables, multivariate regression models adjusted by the other variables revealed PT angle to be an independent factor for the sum of the total LPM CSA $(\beta=-0.610$, $\left.\mathrm{p}=0.021, \mathrm{R}^{2}=0.320\right)($ Table 4$)$.

\section{DISCUSSION}

The most important finding of this study was that only PT angle was significantly correlated with the sum of the total LPM CSA in healthy older women. Because we used multivariate regression models adjusted for potential factors, including age and ASM, SSB parameters might be independent factors affecting spinal sarcopenia.

In the current study, conventional sarcopenic indices (ASM, HGS, and gait speed) were not correlated with LPM mass, back extensor strength, and spine specific functional outcomes. Even ASM was not correlated with LPM CSA $(r=0.181, p=0.535)$. Therefore, conventional muscle mass measurements that sum limb muscle masses to define sarcopenia do not reflect the clinical features of spinal sarcopenia. Jeon et al. ${ }^{25)}$ reported that low limb muscle mass was correlated with only knee joint radiological degeneration and not hip or spine. Therefore, site-specific muscle mass investigation is necessary to evaluate the effect of skeletal muscle on specific regions.

Among the several SSB parameters, only PT angle was significantly correlated with both LPM quantity (CSA) and quality (density), while LL angle was only correlated with LPM quantity. PT is the angle between a vertical line originating at the center of the femoral head and a line starting from the center of the femoral head to the midpoint of the endplate of S1. In simple terms, this angle describes the rotation of the pelvis around the femoral heads. $\mathrm{PT}$ increases with age, and high PT is needed to maintain an up-

Table 1. Baseline characteristics of the 24 older women

\begin{tabular}{|c|c|}
\hline Characteristic & Value \\
\hline Age (y) & $76.8 \pm 4.2$ \\
\hline Body mass index $\left(\mathrm{kg} / \mathrm{m}^{2}\right)$ & $24.9 \pm 2.4$ \\
\hline \multicolumn{2}{|l|}{ Conventional sarcopenic indices } \\
\hline $\operatorname{ASM}(\mathrm{kg})$ & $14.6 \pm 2.3$ \\
\hline $\operatorname{ASM} / \mathrm{Ht}^{2}\left(\mathrm{~kg} / \mathrm{m}^{2}\right)$ & $6.30 \pm 0.79$ \\
\hline Gait speed $(\mathrm{m} / \mathrm{s})$ & $0.87 \pm 0.16$ \\
\hline Handgrip strength (kg) & $20.0 \pm 3.4$ \\
\hline \multicolumn{2}{|l|}{ Other functional test } \\
\hline Back performance scale & $2.96 \pm 1.97$ \\
\hline Oswestry Disability Index & $8.33 \pm 5.84$ \\
\hline Short physical performance battery & $10.4 \pm 1.7$ \\
\hline Timed Up and Go test (s) & $9.4 \pm 2.8$ \\
\hline \multicolumn{2}{|l|}{ Spinopelvic parameters } \\
\hline Sacral slope $\left(^{\circ}\right)$ & $32.9 \pm 11.6$ \\
\hline Pelvic incidence $\left({ }^{\circ}\right)$ & $50.5 \pm 12.8$ \\
\hline Pelvic tilt $\left(^{\circ}\right)$ & $21.5 \pm 7.2$ \\
\hline Lumbar lordosis $\left(^{\circ}\right)$ & $42.2 \pm 11.3$ \\
\hline Thoracic kyphosis $\left(^{\circ}\right)$ & $39.1 \pm 11.2$ \\
\hline Sagittal vertical axis (mm) & $31.8 \pm 33.0$ \\
\hline \multicolumn{2}{|l|}{ Back muscle strength and CT scan } \\
\hline Isometric back extensor strength $(\mathrm{N})$ & $39.8 \pm 12.6$ \\
\hline Multifidus CSA $\left(\mathrm{mm}^{2}\right)$ & $3,514.0 \pm 619.8$ \\
\hline Multifidus density (HU) & $10.2 \pm 13.6$ \\
\hline Erector spinae CSA $\left(\mathrm{mm}^{2}\right)$ & $8,806.8 \pm 1,470.2$ \\
\hline Erector spinae density (HU) & $15.8 \pm 12.4$ \\
\hline Sum of total LPM CSA $\left(\mathrm{mm}^{2}\right)$ & $12,320.8 \pm 1,571.0$ \\
\hline Mean of total LPM density (HU) & $14.3 \pm 11.7$ \\
\hline
\end{tabular}

Values are presented as mean \pm standard deviation.

ASM, appendicular skeletal muscle mass; CSA, cross-sectional area; CT, computed tomography; $\mathrm{Ht}^{2}$, height squared; LPM, lumbar paraspinal muscle. 

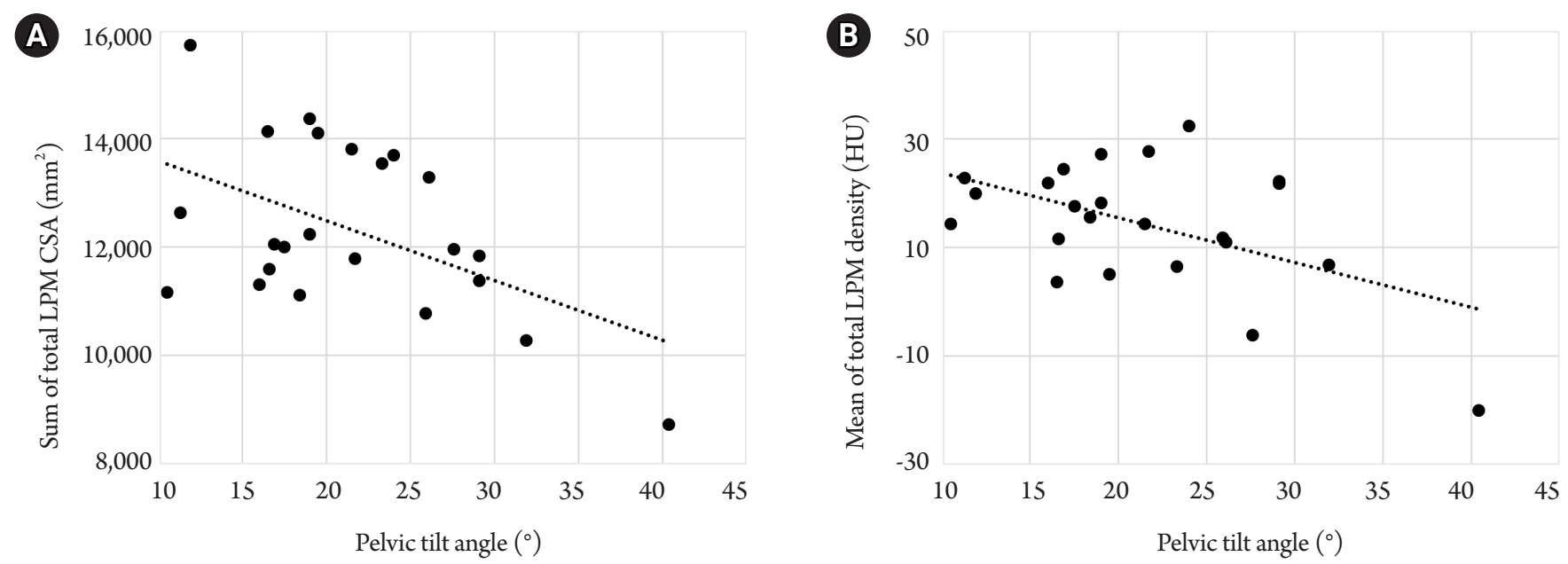

Fig. 2. Scatter grams showing the relationships between pelvic tilt angle and LPM CSA (A) and mean density (B). LPM, lumbar paraspinal muscle; CSA, cross-sectional area.

Table 2. Correlations between sarcopenic indices and functional outcomes

\begin{tabular}{lccccc}
\hline & Age & BMI & ASM/Ht & Gait speed & Handgrip strength \\
\hline SPPB & 0.063 & -0.243 & -0.241 & $0.599^{*}$ & $0.521^{*}$ \\
TUG & -0.014 & $0.415^{*}$ & 0.458 & -0.369 & -0.296 \\
ODI & -0.064 & 0.047 & 0.341 & -0.170 & -0.099 \\
BPS & 0.171 & $0.502^{*}$ & 0.288 & -0.250 & -0.307 \\
Sum of total LPM CSA & -0.220 & 0.158 & 0.181 & -0.272 & -0.017 \\
Mean of total LPM density & -0.099 & -0.005 & 0.159 & 0.078 & 0.076 \\
Back extensor strength & -0.321 & 0.310 & 0.207 & -0.058 & 0.366 \\
\hline
\end{tabular}

BMI, body mass index; ASM, appendicular skeletal muscle mass; $\mathrm{Ht}^{2}$, height squared; CSA, cross-sectional area; SPPB, short physical performance battery; TUG, Timed Up and Go test; ODI, Oswestry Disability Index; BPS, Back Performance Scale; LPM, lumbar paraspinal muscle.

${ }^{*} \mathrm{p}<0.05$ by Pearson correlation coefficient.

Table 3. Correlations between spinal sagittal balance indices and functional outcomes

\begin{tabular}{lcccccc}
\hline & LL & TK & PT & SS & PI & SVA \\
\hline SPPB & -0.028 & -0.021 & 0.015 & -0.158 & -0.121 & -0.084 \\
TUG & -0.010 & 0.030 & 0.170 & 0.046 & 0.097 & 0.042 \\
ODI & $-0.423^{*}$ & $-0.414^{*}$ & 0.169 & -0.109 & -0.010 & 0.165 \\
BPS & 0.128 & -0.046 & 0.026 & 0.100 & 0.013 & -0.251 \\
Sum of total LPM CSA & $0.439^{*}$ & 0.399 & $-0.502^{*}$ & -0.080 & -0.299 & -0.394 \\
Mean of total LPM density & 0.319 & 0.388 & $-0.504^{*}$ & 0.335 & -0.036 & -0.221 \\
Back extensor strength & -0.161 & 0.285 & -0.087 & -0.385 & $-0.490^{*}$ & -0.110 \\
\hline
\end{tabular}

LL, lumbar lordosis; TK, thoracic kyphosis; PT, pelvic tilt; SS, sacral slope; PI, pelvic incidence; SVA, sagittal vertical axis; SPPB, short physical performance battery; TUG, Timed Up and Go test; ODI, Oswestry Disability Index; BPS, Back Performance Scale; LPM, lumbar paraspinal muscle; CSA, cross-sectional area.

${ }^{*} \mathrm{p}<0.05$ by Pearson correlation coefficient.

Table 4. Multivariate regression analysis

\begin{tabular}{lcccccc}
\hline Predictor & Standard error & Standardized coefficients & $\mathrm{t}$ & $\mathrm{p}$-value & $\mathrm{R}$ & Adjusted R $^{2}$ \\
\hline (Constant) & 1070.403 & - & 13.857 & 0.000 & - & - \\
Pelvic tilt & 51.061 & -0.610 & -0.266 & 0.021 & 0.610 & 0.320 \\
\hline
\end{tabular}


right posture to compensate for kyphosis. ${ }^{26)}$ While other SSB parameters such as LL and SVA can be easily affected by posture and position, The PT is a reproducible and reliable measure of global sagittal alignment regardless of the level of training. ${ }^{27,28)} \mathrm{PT}$ is also correlated with health-related quality of life in adult spinal deformity. ${ }^{29)}$ Therefore, among the SSB parameters, the association of PT with spinal sarcopenia warrants further investigation.

Isokinetic back muscle strength positively affects SSB ${ }^{24)} \mathrm{A}$ cohort study in older adults also reported the negative correlation between spinal inclination and back muscle strength $(r=-0.294) .{ }^{30}$ However, in our study, back extensor strength was not independently associated with SSB, contrary to the hypothesis, although there was a simple correlation between back extensor and PI $(r=-0.490)$. There are two potential explanation for this conflict. If the values of back extensor strengths measured in this study were normal because participants were healthy community-dwelling older women without sarcopenia, they might not affect SSB due to the ceiling effect. Another assumption was that the muscle strength measured in this study was the isometric back extensor strength, which might not be valid in older adults. Therefore, back extensor strength might be better to be evaluated using the gold-standard isokinetic dynamometer.

Our study had several limitations. First, this was a cross-sectional study and not a prospective investigation. In addition, the sample size $(n=24)$ was not sufficient for a good prediction level in the regression model. ${ }^{31)}$ Thus, the longitudinal SSB effects on spinal sarcopenia and causal relationship between SSB and spinal sarcopenia could not be verified. However, we will prospectively follow-up and evaluate the participants to answer these questions in a future study. Second, bias was possible in the participant selection. Because we enrolled only healthy and community-dwelling older women, there were no women with sarcopenia. Therefore, even though we compared sarcopenic indices to functional outcomes and SSB parameters, these outcome variables might be skewed to a healthy population and might not reflect sarcopenia and paraspinal muscle degeneration. Recently, Ohyama et al. ${ }^{32)}$ reported the relationship between sarcopenia and spinopelvic parameters in 126 participants, $21.4 \%$ of whom were patients with sarcopenia. The authors reported larger SVA and TK in the sarcopenia group than those in the group without sarcopenia among patients with a spinopelvic mismatch. Thus, participants diagnosed with sarcopenia should be sufficiently included in the target population. Finally, we did not investigate the global alignment and proportion (GAP) score, which can denote 'normal' and 'pathologic' standing sagittal alignment and shape as a single score for every magnitude of pelvic incidence. ${ }^{33)}$ In the GAP, the optimal sagittal alignment is based on four factors deviating from their ideal curves and these factors are proportionally related to the PI. ${ }^{34)}$ Therefore, future studies should describe the SSB by measuring a single variable, such as the GAP score, rather than listing several different variables.

In conclusion, our data suggest PT angle was significantly correlated with LPM CSA in healthy older women. To our knowledge, this is the first report to investigate the relationships between sarcopenic indices and spinal muscle degeneration with SSB.

\section{CONFLICT OF INTEREST DISCLOSURES}

The researchers claim no conflicts of interest.

\section{ACKNOWLEDGEMENTS}

This research was funded by the Korean Geriatrics Society.

\section{REFERENCES}

1. Masaki M, Ikezoe T, Fukumoto Y, Minami S, Tsukagoshi R, Sakuma K, et al. Association of sagittal spinal alignment with thickness and echo intensity of lumbar back muscles in middle-aged and elderly women. Arch Gerontol Geriatr 2015;61:197-201.

2. Lee SY, Ro HJ, Chung SG, Kang SH, Seo KM, Kim DK. Low skeletal muscle mass in the lower limbs is independently associated to knee osteoarthritis. PLoS One 2016;11:e0166385.

3. Hikata T, Watanabe K, Fujita N, Iwanami A, Hosogane N, Ishii $\mathrm{K}$, et al. Impact of sagittal spinopelvic alignment on clinical outcomes after decompression surgery for lumbar spinal canal stenosis without coronal imbalance. J Neurosurg Spine 2015;23:451-8.

4. Beyer F, Geier F, Bredow J, Oppermann J, Eysel P, Sobottke R. Influence of spinopelvic parameters on non-operative treatment of lumbar spinal stenosis. Technol Health Care 2015;23:871-9.

5. Sinaki M, Itoi E, Rogers JW, Bergstralh EJ, Wahner HW. Correlation of back extensor strength with thoracic kyphosis and lumbar lordosis in estrogen-deficient women. Am J Phys Med Rehabil 1996;75:370-4.

6. Zhu Z, Xu L, Zhu F, Jiang L, Wang Z, Liu Z, et al. Sagittal alignment of spine and pelvis in asymptomatic adults: norms in Chinese populations. Spine (Phila Pa 1976) 2014;39:E1-6.

7. Gelb DE, Lenke LG, Bridwell KH, Blanke K, McEnery KW. An analysis of sagittal spinal alignment in 100 asymptomatic middle and older aged volunteers. Spine (Phila Pa 1976) 1995;20:1351-8.

8. Meakin JR, Fulford J, Seymour R, Welsman JR, Knapp KM. The relationship between sagittal curvature and extensor mus- 
cle volume in the lumbar spine. J Anat 2013;222:608-14.

9. Baumgartner RN, Koehler KM, Gallagher D, Romero L, Heymsfield SB, Ross RR, et al. Epidemiology of sarcopenia among the elderly in New Mexico. Am J Epidemiol 1998; 147:755-63.

10. Pedrero-Chamizo R, Albers U, Tobaruela JL, Melendez A, Castillo MJ, Gonzalez-Gross M. Physical strength is associated with Mini-Mental State Examination scores in Spanish institutionalized elderly. Geriatr Gerontol Int 2013;13:1026-34.

11. Ro HJ, Kim DK, Lee SY, Seo KM, Kang SH, Suh HC. Relationship between respiratory muscle strength and conventional sarcopenic indices in young adults: a preliminary study. Ann Rehabil Med 2015;39:880-7.

12. Chen LK, Liu LK, Woo J, Assantachai P, Auyeung TW, Bahyah KS, et al. Sarcopenia in Asia: consensus report of the Asian Working Group for Sarcopenia. J Am Med Dir Assoc 2014;15:95-101.

13. Guralnik JM, Ferrucci L, Simonsick EM, Salive ME, Wallace RB. Lower-extremity function in persons over the age of 70 years as a predictor of subsequent disability. N Engl J Med 1995;332:556-61.

14. Shumway-Cook A, Brauer S, Woollacott M. Predicting the probability for falls in community-dwelling older adults using the Timed Up \& Go Test. Phys Ther 2000;80:896-903.

15. Ortega-Perez de Villar L, Martinez-Olmos FJ, Junque-Jimenez A, Amer-Cuenca JJ, Martinez-Gramage J, Mercer T, et al. Test-retest reliability and minimal detectable change scores for the short physical performance battery, one-legged standing test and timed up and go test in patients undergoing hemodialysis. PLoS One 2018; 13:e0201035.

16. Kim DY, Lee SH, Lee HY, Lee HJ, Chang SB, Chung SK, et al. Validation of the Korean version of the Oswestry Disability Index. Spine (Phila Pa 1976) 2005;30:E123-7.

17. Strand LI, Moe-Nilssen R, Ljunggren AE. Back Performance Scale for the assessment of mobility-related activities in people with back pain. Phys Ther 2002;82:1213-23.

18. Harding AT, Weeks BK, Horan SA, Little A, Watson SL, Beck BR. Validity and test-retest reliability of a novel simple back extensor muscle strength test. SAGE Open Med 2017;5: 2050312116688842.

19. Stark T, Walker B, Phillips JK, Fejer R, Beck R. Hand-held dynamometry correlation with the gold standard isokinetic dynamometry: a systematic review. PM R 2011;3:472-9.

20. Vialle R, Levassor N, Rillardon L, Templier A, Skalli W, Guigui P. Radiographic analysis of the sagittal alignment and balance of the spine in asymptomatic subjects. J Bone Joint Surg Am 2005; 87:260-7.
21. Buckland AJ, Ramchandran S, Day L, Bess S, Protopsaltis T, Passias PG, et al. Radiological lumbar stenosis severity predicts worsening sagittal malalignment on full-body standing stereoradiographs. Spine J 2017;17:1601-10.

22. Lee SH, Park SW, Kim YB, Nam TK, Lee YS. The fatty degeneration of lumbar paraspinal muscles on computed tomography scan according to age and disc level. Spine J 2017;17:81-7.

23. Sasaki T, Yoshimura N, Hashizume H, Yamada H, Oka H, Matsudaira K, et al. MRI-defined paraspinal muscle morphology in Japanese population: the Wakayama Spine Study. PLoS One 2017;12:e0187765.

24. Hirano K, Imagama S, Hasegawa Y, Wakao N, Muramoto A, Ishiguro N. Effect of back muscle strength and sagittal spinal imbalance on locomotive syndrome in Japanese men. Orthopedics 2012;35:e1073-8.

25. Jeon H, Lee SU, Lim JY, Chung SG, Lee SJ, Lee SY. Low skeletal muscle mass and radiographic osteoarthritis in knee, hip, and lumbar spine: a cross-sectional study. Aging Clin Exp Res 2019;Jan 7; [Epub]. http://doi.org/10.1007/s40520-0181108-5.

26. Roussouly P, Nnadi C. Sagittal plane deformity: an overview of interpretation and management. Eur Spine J 2010;19:1824-36.

27. Plachta SM, Israel H, Brechbuhler J, Hayes A, Huebner S, Place HM. Inter/intraobserver reliability of T1 pelvic angle (TPA), a novel radiographic measure for global sagittal deformity. Spine (Phila Pa 1976) 2018;43:E1290-E1296.

28. Protopsaltis TS, Lafage R, Smith JS, Passias PG, Shaffrey CI, Kim HJ, et al. The lumbar pelvic angle, the lumbar component of the T1 pelvic angle, correlates with HRQOL, PI-LL mismatch, and it predicts global alignment. Spine (Phila Pa 1976) 2018;43:681-7.

29. Lafage V, Schwab F, Patel A, Hawkinson N, Farcy JP. Pelvic tilt and truncal inclination: two key radiographic parameters in the setting of adults with spinal deformity. Spine (Phila Pa 1976) 2009;34:E599-606.

30. Hirano K, Imagama S, Hasegawa Y, Wakao N, Muramoto A, Ishiguro N. Impact of spinal imbalance and back muscle strength on locomotive syndrome in community-living elderly people. J Orthop Sci 2012;17:532-7.

31. Knofczynski GT, Mundfrom D. Sample sizes when using multiple linear regression for prediction. Educ Psychol Meas 2008; 68(3):431-42.

32. Ohyama S, Hoshino M, Terai H, Toyoda H, Suzuki A, Takahashi S, et al. Sarcopenia is related to spinal sagittal imbalance in patients with spinopelvic mismatch. Eur Spine J 2019;28:192936.

33. Yilgor C, Sogunmez N, Boissiere L, Yavuz Y, Obeid I, Kleinstuck 
148 Dong Hyun Kim et al.

F, et al. Global Alignment and Proportion (GAP) score: development and validation of a new method of analyzing spinopelvic alignment to predict mechanical complications after adult spinal deformity surgery. J Bone Joint Surg Am 2017;99:166172.
34. Jacobs E, van Royen BJ, van Kuijk SM, Merk JM, Stadhouder A, van Rhijn LW, et al. Prediction of mechanical complications in adult spinal deformity surgery-the GAP score versus the Schwab classification. Spine J 2019;19:781-8. 